\title{
PTH Signaling During Exercise Contributes to Bone Adaptation
}

\author{
Joseph D Gardinier, ${ }^{1}$ Fatma Mohamed, ${ }^{2}$ and David H Kohn ${ }^{1,3}$ \\ ${ }^{1}$ Biologic and Materials Sciences, University of Michigan, Ann Arbor MI, USA \\ ${ }^{2}$ Oral Health Sciences Program, University of Michigan, Ann Arbor MI, USA \\ ${ }^{3}$ Biomedical Engineering, University of Michigan, Ann Arbor MI, USA
}

\begin{abstract}
Improving the structural integrity of bone reduces fracture risk and development of osteoporosis later in life. Exercise can increase the mechanical properties of bone, and this increase is often attributed to the dynamic loading created during exercise. However, the increase in systemic parathyroid hormone (PTH) levels during exercise gives reason to hypothesize that PTH signaling also regulates bone adaptation in response to exercise. Therefore, the first aim of this study was to establish the impact PTH signaling has on bone adaptation during exercise by inhibiting PTH signaling with PTH(7-34); the second aim was to determine whether increasing PTH levels during exercise with PTH(1-34) can augment bone adaptation. Thirty minutes after a single bout of running on a treadmill, mice exhibited a twofold increase in systemic PTH levels. Under the same exercise regimen, the influence of PTH signaling on bone adaptation during exercise was then evaluated in mice after 21 consecutive days of exercise and treatment with PTH(7-34), PTH(134), or vehicle. Exercise alone caused a significant increase in trabecular bone volume with adaptation to a more platelike structure, which was inhibited with PTH(7-34) during exercise. Changes in structural-level and tissue-level mechanical properties during exercise occurred in the absence of significant changes to cortical bone geometry. Inhibition of PTH signaling during exercise attenuated the changes in structural-level mechanical properties, but not tissue-level properties. Enhanced PTH signaling during exercise with PTH(1-34) increased trabecular and cortical bone volume, but had little effect on the structural-level and tissue-level mechanical properties compared to exercise alone. Our study is the first to demonstrate that bone adaptation during exercise is not only a function of dynamic loading, but also PTH release, and that PTH signaling contributes differently at the structural and tissue levels. ( 2015 American Society for Bone and Mineral Research.
\end{abstract}

KEY WORDS: EXERCISE; PTH; BIOMECHANICS; ANALYSIS/QUANTITATION OF BONE; ANABOLICS

\section{Introduction}

T he prevalence of osteoporosis has grown over the years; this disease is estimated to have caused over 9 million fractures across the world, many of which cause permanent disabilities or complications resulting in death. ${ }^{(1)}$ Anabolic treatment strategies that enhance the mechanical properties of bone and/or offset the progression of bone loss are highly sought after, but not readily available. ${ }^{(2)}$ Current treatments attempt to improve the mechanical integrity of the skeleton by increasing the quantity of bone or improving its quality, as defined by its strength and toughness. Although exercise has been widely considered a potential treatment strategy to improve the mechanical properties of bone, the specific mechanisms through which mechanical properties are increased during exercise remain unclear. ${ }^{(3)}$ Insight into such mechanisms will contribute to developing treatments that use exercise to improve the integrity of bone or even prevent osteoporosis later in life.

Among humans, both treadmill running and swimming increase bone formation based on increased bone mineral density $(B M D),{ }^{(3)}$ along with serum levels of bone alkaline phosphatase. ${ }^{(4)}$ In rodents, both swimming and treadmill running increase trabecular bone volume and thickness, $(6,7)$ along with significant increases in both tissue-level and structural-level properties of cortical bone. ${ }^{(8-12)}$ The adaptation in structural-level properties of cortical bone is attributed to changes in geometry due to periosteal expansion. ${ }^{(11,13)}$ However, running can also modify the tissue-level properties of cortical bone without altering cross-sectional geometry. ${ }^{(8,10,12)}$ This adaptation in tissue-level properties, independent of geometric changes, has been associated with changes to tissue composition, such as increases in collagen cross-linking and changes in the carbonate/phosphate ratio. ${ }^{(10,14)}$ Overall, literature supports the general concept that moderate exercise improves the mechanical function of bone by modifying either its structural-level or tissue-level mechanical properties.

The dynamic loading incurred during exercise is considered to have the dominant impact on bone adaptation, and can reach magnitudes of 2000 microstrain in humans. ${ }^{(15)}$ Direct loading of the tibia or ulna of rodents under similar magnitudes of strain

Received in original form July 10, 2014; revised form December 3, 2014; accepted December 8, 2014. Accepted manuscript online December $22,2014$. Address correspondence to: David H Kohn, PhD, Dept. of Biological and Material Sciences, 2213 School of Dentistry, University of Michigan, Ann Arbor, MI 48109-1078, USA. E-mail: dhkohn@umich.edu

Journal of Bone and Mineral Research, Vol. 30, No. 6, June 2015, pp 1053-1063

DOI: $10.1002 / j b m r .2432$

(C) 2015 American Society for Bone and Mineral Research 
initiates osteocyte communication with osteoclasts and osteoblasts responsible for bone remodeling, ${ }^{(16-18)}$ along with the ensuing increases in bone strength. ${ }^{(19-22)}$ In addition to the influence dynamic loading has on bone adaptation, exercise also provides other stimuli that play a significant role in adaptation, such as the release of parathyroid hormone (PTH). ${ }^{(23)}$ In both humans and animals, the transient increase in systemic PTH levels is dependent on the type, intensity, and duration of exercise. ${ }^{(4,5,9,23,24)}$ Moderate running among human adults causes a significant increase in systemic levels of PTH at the onset of exercise that remain high 1 to 2 hours after exercise has stopped, then return to baseline. ${ }^{(4,24)}$ Transient increases in systemic PTH during exercise may be analogous to daily treatments of PTH, which over the course of several weeks increase trabecular bone volume along with cortical bone volume and modulus of elasticity. ${ }^{(25)}$ Given that PTH treatment can further increase cortical bone formation achieved under direct loading. ${ }^{(25,26)}$ PTH signaling during exercise has the potential to synergistically influence bone formation and the adaptation of its mechanical properties.

The purpose of this study, therefore, was (1) to establish the impact PTH release has on bone adaptation during exercise by inhibiting the signaling mechanism with PTH(7-34), and (2) to augment bone adaptation by increasing the PTH levels during exercise with PTH(1-34). We hypothesized that inhibiting PTH signaling during exercise would inhibit trabecular bone formation along with inhibiting increases to the structural-level properties of cortical bone, and that increasing the PTH levels with $\mathrm{PTH}(1-34)$ would increase bone formation and increase its mechanical properties at the structural level.

\section{Materials and Methods}

\section{In vivo protocols}

Animal procedures were performed at the University of Michigan with the approval of the University Committee on Use and Care of Animals (UCUCA). Male C57BI/6J mice (16 weeks old at the start of each experiment) purchased from Jackson Laboratories (Bar Harbor, ME, USA), were individually housed throughout the entire study (because of their aggressive nature) with sufficient water, food, and items for enrichment.

\section{Exercise effects on systemic PTH levels}

To establish the effect a single bout of exercise has on systemic PTH levels, 50 mice were first subjected to exercise that involved moderate running on a treadmill (Columbus Instruments, $\mathrm{OH}$, USA) with a 5-degree incline for 30 minutes/day at speeds increasing from 8 to $12 \mathrm{~m} / \mathrm{min}$ over the course of 3 days. After 3 days of training, the mice were assigned to weight-matched groups: baseline or exercise. The exercise group was then divided into five subgroups, each group representing a different time point at which blood samples were taken: $0,1,1.5,3$, and 24 hours after the onset of exercise. At each time point, blood samples were also taken from mice in the baseline group, which did not exercise on day 4 . For each mouse, $\sim 100 \mu \mathrm{L}$ of blood was drawn from the submandibular vein into a centrifuge tube with an anticoagulate and then immediately spun down at $1000 \mathrm{~g}$ at $8^{\circ} \mathrm{C}$ for $15 \mathrm{~min}$. The plasma was immediately separated and stored at $-80^{\circ} \mathrm{C}$ for later processing. Plasma samples were prepared and analyzed using the PTH ELISA Kit (Immutopics International) according to the manufacturer's protocol on a microplate reader (Varioskan Flash; Thermo Scientific).
Efficacy of PTH(7-34) for inhibiting PTH/PTH-related protein receptor

The efficacy of PTH(7-34) to inhibit activation of the PTH/PTHrelated protein receptor (PPR) was established based on its ability to inhibit changes in c-Fos gene expression regulated downstream of the PPR. Given that PTH(1-34) activation of the PPR increases c-Fos expression among osteoblasts and osteocytes, ${ }^{(27)}$ mice were first treated with PTH(7-34) and then PTH(134) either 1 hour or 3 hours later. A total of 35 mice were divided into five groups: vehicle, PTH(7-34), PTH(1-34), PTH(7-34) + PTH$(1-34)_{1 \mathrm{hr}}$, and PTH(7-34) $+\mathrm{PTH}(1-34)_{3 \mathrm{hr}}$. Subcutaneous injections were given to administer $50 \mu \mathrm{L}$ of saline solution $(0.9 \% \mathrm{NaCl})$ as a vehicle, $60 \mu \mathrm{g} / \mathrm{kg}$ (body weight) of (d-Try12, Try34)-bPTH(7-34) (Bachem, CA, USA) in saline solution, or $40 \mu \mathrm{g} / \mathrm{kg}$ of $\mathrm{hPTH}(1-34)$ (Bachem) in saline solution. After the final injection, mice were euthanized via $\mathrm{CO}_{2}$ asphyxiation, and the tibia were extracted, cleaned of excess soft tissue, and then homogenized to extract the mRNA using a Trizol method. The mRNA was purified before generating cDNA (TaqMan cDNA synthesis kit; Applied Biosystems). The qRT-PCR was carried out with an Applied Biosystems 7500 RealTime PCR machine along with primers for c-Fos (Mm00487425; TaqMan) and GAPDH as an internal control (Mm99999915, TaqMan). The standard curve method was used to determine gene expression for each sample relative to GAPDH levels, and then reported as a fold change compared to vehicle-treated mice.

\section{Exercise effects on bone adaptation}

To determine the influence PTH signaling during exercise has on bone adaptation, 105 male mice were assigned to one of seven weight-matched groups: three sedentary groups, three exercise groups, and a baseline group. The exercise groups were subjected to treadmill running at $12 \mathrm{~m} / \mathrm{min}$ on a 5 degree incline for $30 \mathrm{~min}$ each day over 21 consecutive days. Each exercise group received daily subcutaneous injections of either $50 \mu \mathrm{L}$ of saline solution $(0.9 \% \mathrm{NaCl})$ as a vehicle, $60 \mu \mathrm{g} / \mathrm{kg}$ (body weight) of (d-Try12, Try34)-bPTH(7-34) (Bachem) in saline solution, or $40 \mu \mathrm{g} / \mathrm{kg}$ of $\mathrm{hPTH}(1-34)$ (Bachem) in saline solution. Both vehicle and PTH(7-34) treatments were administered 3 hours prior to exercise, whereas PTH(1-34) was administered 15 min prior to exercise. Each sedentary group was also treated with either vehicle, PTH(7-34), or PTH(1-34) over the course of 21 days.

To quantify bone formation histomorphometrically, each mouse was also given an intraperitoneal (i.p.) injection of Alizarin Red $(25 \mathrm{mg} / \mathrm{kg}$ body weight) on day 3, and an i.p. injection of Xylene Orange ( $90 \mathrm{mg} / \mathrm{kg}$ body weight) on day 15 . Each mouse was then euthanized on day 22, and both tibias were extracted and stored at $-20^{\circ} \mathrm{C}$ wrapped in gauze soaked with $1 \times$ phosphate buffered saline (PBS) $+57 \mathrm{mg} / \mathrm{L}$ of calcium. One tibia from each mouse was selected for micro-computed tomography $(\mu \mathrm{CT})$ analysis and mechanical testing, whereas the contralateral tibia was used for histomorphometry.

\section{$\mu C T$}

Tibias were scanned prior to mechanical testing in a Scanco $\mu \mathrm{CT}$ system ( $\mu \mathrm{CT} 100$; Scanco Medical, Bassersdorf, Switzerland) with the following settings: $12 \mu \mathrm{m}$ voxel size, medium resolution, 70 $\mathrm{kVp}, 114 \mu \mathrm{A}, 0.5 \mathrm{~mm}$ aluminum filter, and $500 \mathrm{~ms}$ integration time. Image slices were processed with a grayscale threshold optimized between 0 and 1000 across a population of samples. 
For each sample, a $500-\mu \mathrm{m}$-thick volume of interest, $50 \mu \mathrm{m}$ below the proximal growth plate, was selected for threedimensional (3D) analysis of the trabecular bone structure. Based on the direct 3D method provided in the manufacturer's software, the following trabecular parameters were calculated: bone volume fraction (BV/TV), tissue mineral density (TMD), trabecular number (Tb.N), trabecular spacing (Tb.Sp), trabecular thickness (Tb.Th), and structural model index (SMI). To determine the degree of anisotropy (directional dependence) of the trabeculae, eigenvalues of the fabric tensor were calculated based on the mean intercept length technique, and normalized to obtain $\mathrm{H}_{1}, \mathrm{H}_{2}$, and $\mathrm{H}_{3}$ along with the angle between the principal vector and the tibia's longitudinal axis (theta). ${ }^{(28)}$ The degree of anisotropy (DA) of the trabeculae was defined by the ratio between the largest and smallest vectors of the normalized eigenvalues.

Once each tibia was scanned and mechanically tested (see Mechanical testing, below), images of each tibia were first rotated to match their position during mechanical testing. The cortical bone thickness (Ct.Th), cortical cross-sectional area (Ct. $\mathrm{Ar})$, moment of inertia about the anterior-posterior axis $\left(\mathrm{I}_{\mathrm{ap}}\right)$, moment of inertia about the medial-lateral axis $\left(I_{\mathrm{ml}}\right)$, distance from the most lateral surface to the neutral axis $\left(D_{N-A}\right)$, bone mineral content (BMC), and BMD were determined across an 180- $\mu \mathrm{m}$-thick section at the fracture site and a standard site. The fracture site was defined where failure was initiated during mechanical testing, whereas the standard site was defined midway between the loading points.

\section{Mechanical testing}

Structural-level and tissue-level properties of each tibia scanned for $\mu C T$ analysis were determined under four-point bending using an Admet eXpert 450 Universal Testing Machine. ${ }^{(11)}$ The base support span was $9 \mathrm{~mm}$ with a loading span of $3 \mathrm{~mm}$. The tibia was positioned in the loading device such that the lateral surface was in compression by placing the most distal portion of the tibia and fibula junction (TFJ) directly over the leftmost support point. Each tibia was loaded at a rate of $0.01 \mathrm{~mm} / \mathrm{s}$ until failure, while the load and displacement were recorded, which were then used to calculate the tissue-level properties based on beam-bending theory.

\section{Histomorphometry}

Contralateral tibias from the 21-day experiment were first dehydrated in graded ethanol concentrations $(70 \%, 80 \%$, and $100 \%$ ) and then embedded in methyl methacrylate (Koldmount Cold Mount kit; Mager Scientific, MI). Sections 1 to $2 \mathrm{~mm}$ thick were cut at the mid-diaphysis with a diamond wafering blade (Mager Scientific, MI, USA) on a low-speed sectioning saw (South Bay Technology, CA; Model 650) and then polished to a final thickness between 50 and $70 \mu \mathrm{m}$ on wet silicon carbide abrasive disks. A fluorescence microscope was used to identify tissue surfaces labeled red (Alizarin Red) and orange (xylene). The distance between adjacent labels was quantified to determine the mineralizing surfaces (MS), mineral apposition rate (MAR), and bone formation rate (BFR) along the endosteum and periosteum according to standardized histomorphometric analysis. ${ }^{(29)}$

\section{Plasma PTH Levels During Exercise}

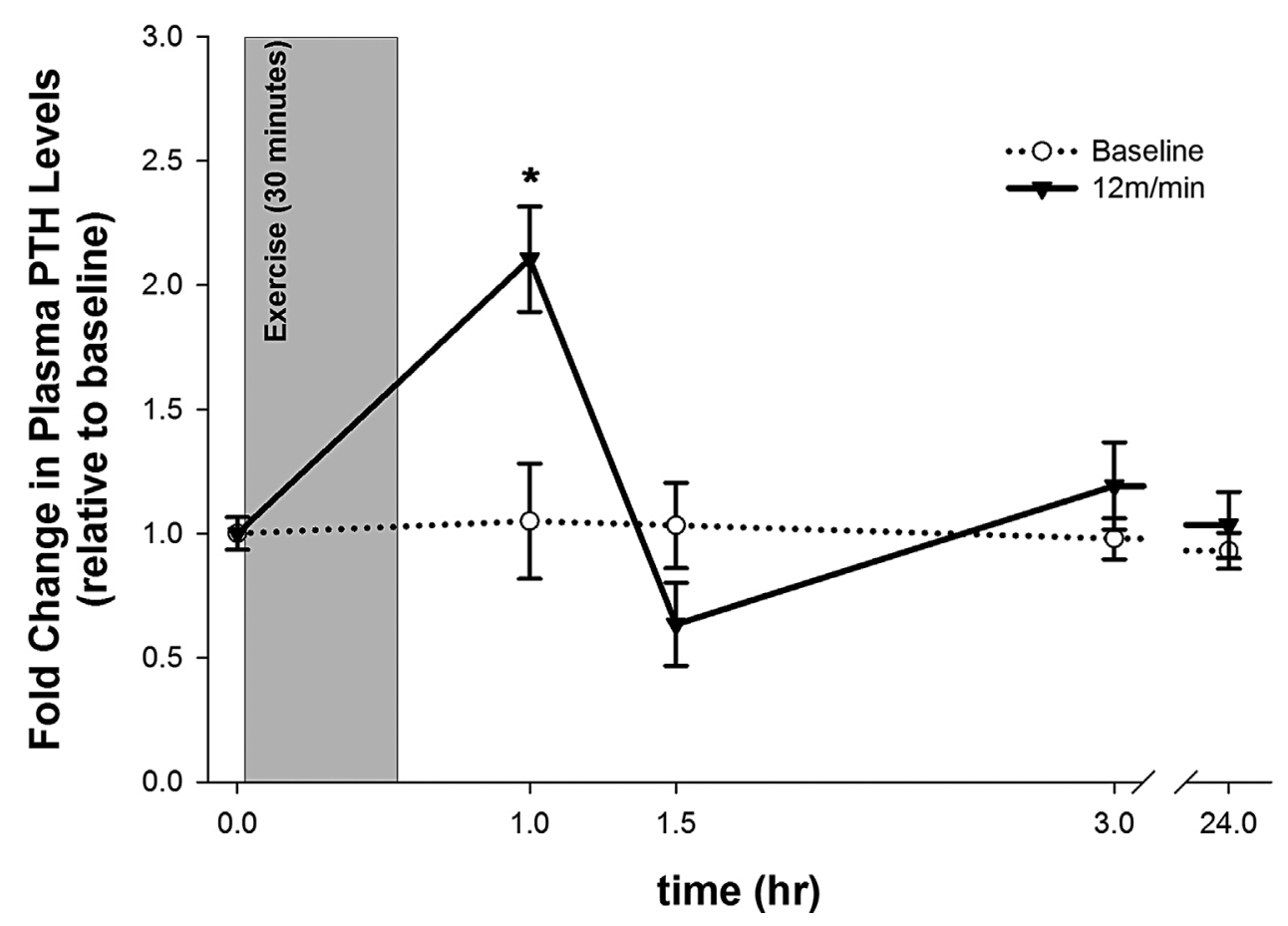

Fig. 1. Plasma levels of PTH were measured in response to a single bout of exercise involving running on a treadmill at $12 \mathrm{~m} / \mathrm{min}$. The average PTH concentration measured at each time point was normalized to the baseline average taken prior to exercise (time $=0$ ). Plasma PTH levels were significantly higher within $30 \mathrm{~min}$ following exercise compared to sedentary controls. Mean \pm SEM ( $n=10,{ }^{*} p<0.05$ compared to baseline timepoint). 


\section{Statistical analysis}

Paired $t$ tests were used to determine significant changes in body weight from day 1 to day 21 of the exercise program, with a $p$ value $<0.05$ denoting significance. All other outcomes were tested for statistical differences between groups in SPSS using a two-way ANOVA for effects of exercise and PTH, with a $p$ value $<0.05$ indicating a significant difference. A Student-Newman Keuls post hoc test was used to test specific interactions between all three sedentary groups, or all three exercise groups, as well as interactions between exercise and sedentary groups of each individual treatment.

\section{Results}

\section{Exercise increases systemic PTH levels}

When subjected to a single bout of exercise, mice exhibited a twofold increase in systemic PTH levels compared to basal levels within 1 hour after the onset of exercise $(333 \pm 49 \mathrm{pg} / \mathrm{mL}$ versus $175 \pm 18 \mathrm{pg} / \mathrm{mL} ; p=0.017$ ) (Fig. 1). The systemic PTH levels returned to basal levels at 1.5 hours after the onset of exercise and remained at basal levels thereafter.

\section{PTH(7-34) inhibits cellular response to PTH in bone}

The ability to inhibit the cellular response to PTH within bone using PTH(7-34) was then established based on c-Fos mRNA expression in response to PTH(1-34). Treatment with PTH(1-34) alone caused a significant increase in c-Fos expression (Fig. 2), similar to other studies. ${ }^{(27)}$ Pretreating mice with $\mathrm{PTH}(7-34) 1$ hour or 3 hours prior to treatment with PTH(1-34) significantly inhibited the change in c-Fos expression by $\sim 75 \%$, whereas treatment alone with $\mathrm{PTH}(7-34)$ had no significant effect. As a result, pretreatment with $\mathrm{PTH}(7-34)$ prior to exercise was considered sufficient to inhibit the cellular response to PTH during exercise.

\section{Exercise causes weight loss}

Mice in the exercise groups treated with vehicle, $\mathrm{PTH}(7-34)$, or PTH(1-34) had significant weight loss that averaged $-1.34 \pm 0.16 \mathrm{~g}\left(p<1 \times 10^{-6}\right),-1.65 \pm 0.26 \mathrm{~g}\left(p<1 \times 10^{-7}\right)$, and $-1.22 \pm 0.20 \mathrm{~g}\left(p<7 \times 10^{-6}\right)$, respectively, over the 21 -day course of the experiment. Sedentary mice treated with PTH(734) also exhibited significant weight loss, which averaged $-0.46 \pm 0.22 \mathrm{~g}(p=0.044)$. Sedentary mice treated with vehicle or PTH(1-34) had no significant change in body weight over the 3-week experiment, averaging $-0.20 \pm 0.24 \mathrm{~g}(p=0.4)$ and $0.247 \pm 0.27 \mathrm{~g}(p=0.3)$, respectively.

\section{Exercise increases trabecular bone formation and decreases directionality of trabeculae}

In response to 3 weeks of exercise alone, there was a significant increase in trabecular BV/TV and a significant decrease in SMI compared to sedentary controls (Fig. 3). The decrease in SMI indicates that the trabecular structure is shifted toward a more platelike structure, as opposed to a rodlike structure. The Tb.Th following exercise was significantly larger compared to sedentary controls, whereas Tb.Sp, Tb.N, and TMD were unaffected.

All three eigenvalues of the fabric tensor were unequal, indicating an orthotropic material with the material symmetry predominately aligned with the long axis, represented by $\mathrm{H}_{2}$. Compared to sedentary controls, exercise caused a significant

\section{c-FOS mRNA Expression}



Fig. 2. Pretreatment with $\mathrm{PTH}(7-34)$ inhibits the cellular response to PTH in bone. The cellular response in bone to PTH(1-34) was evaluated based on mRNA expression of c-Fos. Mice treated with $\mathrm{PTH}(1-34)$ alone exhibited a 1.5-fold increase in c-Fos expression 1 hour later. Pretreatment with PTH(7-34) 1 hour and 3 hours prior to PTH(1-34) significantly inhibited the increase in c-Fos expression by $\sim 75 \%$ compared to $\mathrm{PTH}(1-$ 34) treatment alone. Mean $\pm \operatorname{SEM}(n=10$, a indicates $p<0.05$ compared to vehicle treated mice, $\mathrm{b}$ indicates $p<0.05$ compared to mice treated with $\mathrm{PTH}(1-34)$ alone).

decrease in the eigenvalue $\mathrm{H}_{2}$ and significantly increased its angle (theta) with the anatomical long axis of the tibia (Fig. 4). The significant increase in theta of $\mathrm{H}_{2}$ demonstrates less alignment of the trabeculae with the longitudinal axis of the tibia following exercise.

PTH signaling during exercise contributes to trabecular bone adaptation

As hypothesized, PTH(7-34) treatment during exercise resulted in a significantly lower trabecular BV/TV compared to exercise alone, as well as a significantly greater SMI (Fig. 3). The normalized eigenvector $\mathrm{H}_{2}$ was also significantly greater in magnitude, with a smaller angle theta compared to vehicle treatment during exercise (Fig. 4).

PTH(1-34) treatment superposed with sedentary and exercise conditions caused a significantly greater Tb.Th, Tb.Sp, and significantly smaller Tb.N compared to the respective vehicletreated controls (Fig. 3). The trabecular BV/TV was statistically greater in sedentary mice treated with PTH(1-34) compared to vehicle treatment, but was not statistically different between exercise groups treated with vehicle or PTH(1-34). Superimposing $\mathrm{PTH}(1-34)$ treatment with exercise significantly increased the trabecular TMD compared to sedentary mice treated with PTH(1-34). The degree of anisotropy was 
A

BV/TV

口Sedentary Exercise

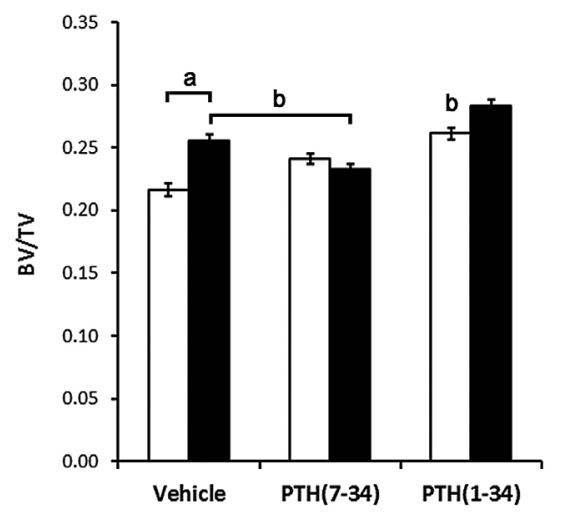

B

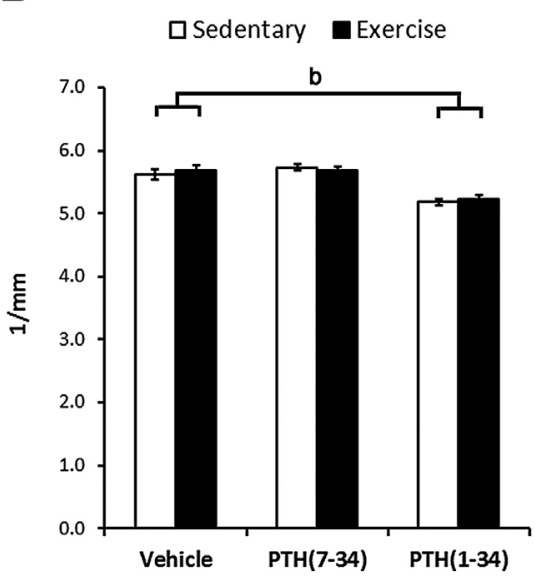

SMI

$\square$ Sedentary Exercise

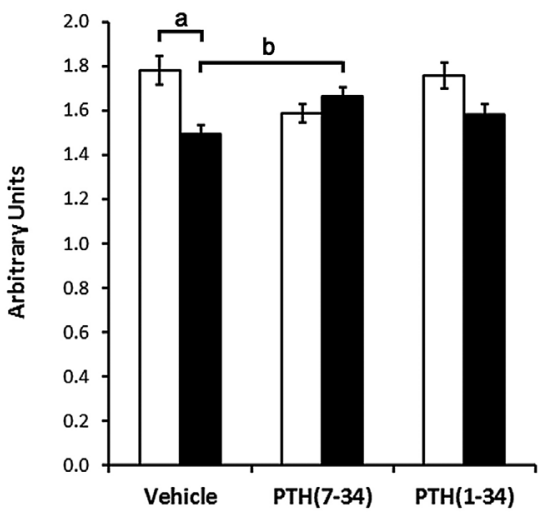

Tb.Th

口Sedentary Exercise

b

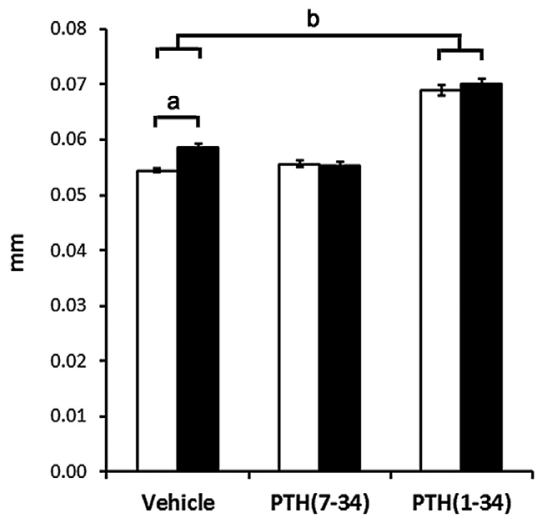

TMD

$\square$ Sedentary Exercise

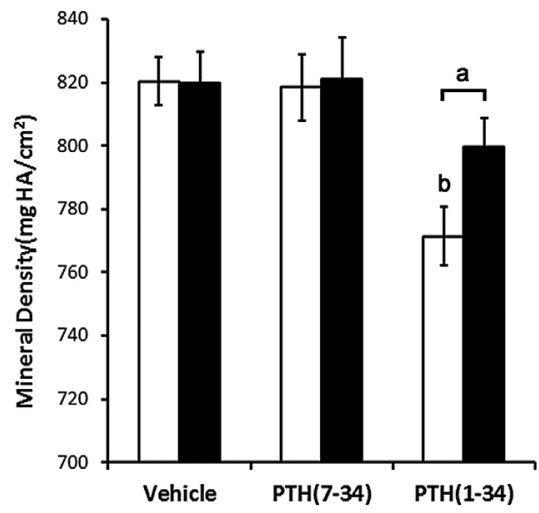

Tb.Sp

$\square$ Sedentary Exercise

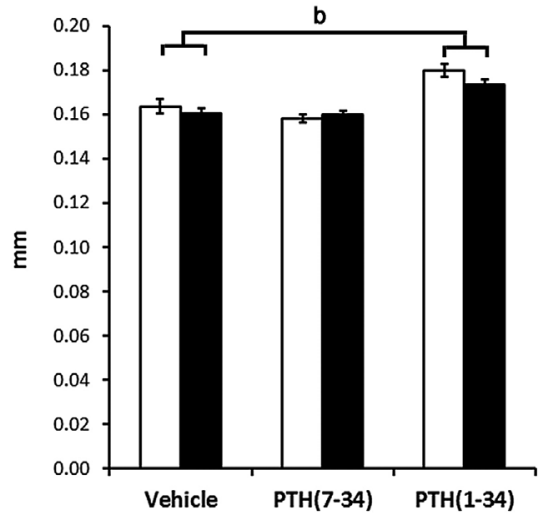

Fig. 3. PTH signaling during exercise contributes to the increase in trabecular BV/TV and decrease in SMI. (A) The BV/TV after exercise, as well as after PTH(1-34) treatment alone, was statistically greater than BV/TV in sedentary mice treated with vehicle alone. The decrease in trabecular SMI due to exercise indicated a more platelike structure, which was inhibited by PTH(7-34) during exercise, but not altered by PTH(1-34) in sedentary or exercise conditions. (B) PTH(1-34) treatment in sedentary and exercise conditions decreased the trabecular number and increased trabecular thickness and spacing with respect to their vehicle-treated controls. Mean $\pm \operatorname{SEM}(n=12$, a indicates $p<0.05$ compared to sedentary control, $\mathrm{b}$ indicates $p<0.05$ compared to vehicle control). $\mathrm{BV} / \mathrm{TV}=$ bone volume fraction; $\mathrm{SMI}=$ structural model index; $\mathrm{TMD}=$ tissue mineral density; $\mathrm{Tb} . \mathrm{N}=$ trabecular number; $\mathrm{Tb}$. $\mathrm{Th}=$ trabecular thickness; $\mathrm{Tb} . \mathrm{Sp}=$ trabecular spacing.

significantly reduced by $\mathrm{PTH}(1-34)$ treatment in both sedentary and exercise conditions compared to the respective vehicletreated controls (Fig. 3). PTH(1-34) treatment of sedentary mice also significantly decreased the eigenvalue $\mathrm{H}_{2}$ and increased its angle theta. Given the inhibitory and additive effect that PTH(734) and PTH(1-34) had during exercise, respectively, trabecular bone adaptation during exercise is attributed, at least in part, to PTH signaling.

Cortical bone formation and geometry are increased by PTH(1-34), but not by exercise

Despite the changes in trabecular bone, exercise had no effect on cortical bone formation rate at either the endosteal or periosteal surface (Fig. 5A-H). The only significant effect exercise had on bone histomorphometric indices was a reduction in the mineralization rate along the endosteum (Endo.MAR), but this decrease was not large enough to augment the bone formation rate (Endo.BFR) (Fig. 5A). $\mathrm{PTH}(1-34)$ treatment led to a significantly greater Endo.BFR, due to an increase in Endo.MS, whereas the significant increase in Perio.BFR was due to an increase in Perio.MS and Perio.MAR (Fig. 5B). A similar response to PTH(1-34) was observed in the exercise group; however, the Endo.MS was not significantly greater than in the vehicle-treated exercise controls.

At the standard site, exercise had no significant impact on $\mathrm{Ct}$. $\mathrm{Ar}, \mathrm{Ct}$.Th, $\mathrm{D}_{\mathrm{N}-\mathrm{A}}$, moments of inertia, BMC, or BMD compared to sedentary controls (Table 1). Treatment with PTH(7-34) during exercise or sedentary conditions had no significant impact on cortical bone geometry, BMC, or BMD compared to controls (Table 1). Similar findings were observed at the fracture site (data not shown).

Among sedentary mice, PTH(1-34) treatment increased Ct.Ar, $\mathrm{Ct} . T h$, and BMC at the standard site compared to vehicle-treated 

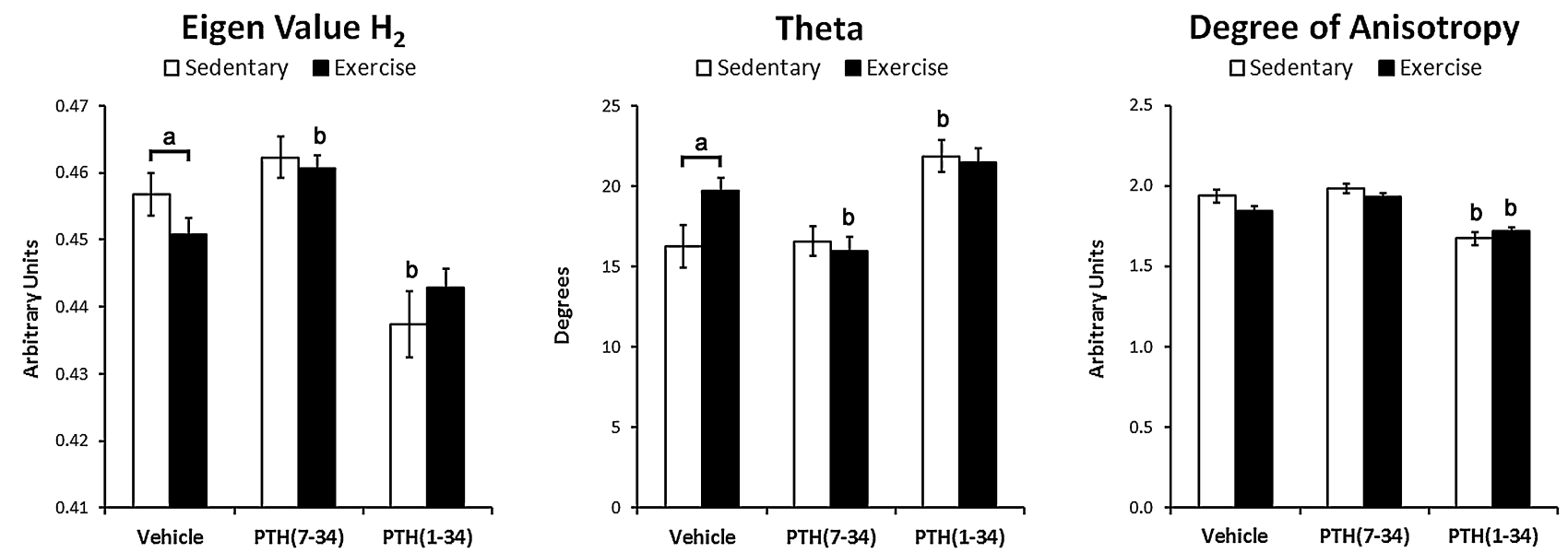

Fig. 4. PTH signaling during exercise regulates trabecular bone orientation. The normalized eigenvalue of $\mathrm{H}_{2}$ was significantly decreased following exercise, whereas the angle between $\mathrm{H}_{2}$ and the tibia's longitudinal axis (theta) was significantly increased. PTH(7-34) treatment during exercise inhibited the decrease in the eigenvalue $\mathrm{H}_{2}$ and the increase in theta. PTH(1-34) treatment in both sedentary and exercise mice caused a significant decrease in the degree of anisotropy, indicating a more isotropic (less directionally dependent) structure. Mean $\pm \operatorname{SEM}(n=12$, a indicates $p<0.05$, b indicates $p<0.05$ compared to vehicle control).

or PTH(7-34)-treated mice (Table 1). The addition of PTH(1-34) treatment during exercise had a similar effect at the standard site, but also caused a significant increase in Ct.Ar, Ct.Th, and $\mathrm{BMC}$ at the fracture site compared to vehicle treatment during exercise (data not shown).

\section{Exercise alters structural-level properties through PTH signaling}

The structural properties of the tibia on which exercise had the largest influence were yield displacement and stiffness (Fig. 6A). The significant increase in stiffness in response to exercise was a result of a significant decrease in the yield displacement, because the yield load was not significantly different compared to sedentary controls. Comparisons across all three exercise and all three sedentary groups demonstrated that exercise had a main effect on work, specifically a significant reduction in preyield work $(2.42 \pm 0.1 \mathrm{~mJ}$ sedentary, $2.13 \pm 0.1 \mathrm{~mJ}$ exercise; $p=0.042$ ) and a significant increase in post-yield work $(2.14 \pm 0.2 \mathrm{~mJ}$ sedentary, $2.85 \pm 0.3 \mathrm{~mJ}$ exercise; $p=0.039)$.

PTH(7-34) treatment reduced the impact of exercise on both yield displacement and stiffness, demonstrating that PTH signaling contributes to the changes in structural-level properties of cortical bone during exercise (Fig. 6A). The addition of PTH(1-34) during exercise did not significantly change any of the structural-level properties compared to exercise controls treated with vehicle, but exhibited a significant decrease in yield displacement compared to sedentary controls treated with PTH(1-34). PTH(1-34) treated sedentary mice had significantly higher stiffness due to an increase in yield load compared to vehicle-treated sedentary mice. Despite the main effect of exercise on pre-yield and post-yield work, no significant interactions were found between the individual groups.

\section{Exercise modifies tissue-level properties independent of PTH signaling}

Despite the absence of changes in cross-sectional geometry or BMD following exercise, at the tissue level, there was a significant increase in ultimate stress and modulus with a significant decrease in yield strain compared to sedentary controls (Fig. 6B). Unlike the structural-level properties, PTH(734) treatment during exercise did not inhibit the significant changes in tissue-level properties, indicating that PTH signaling during exercise does not contribute to the changes in tissuelevel properties.

Similar to the structural-level properties, the addition of PTH(1-34) during exercise had no significant influence on tissuelevel properties compared to exercise mice treated with vehicle or sedentary controls treated with PTH(1-34) (Fig. 6B). Sedentary mice treated with PTH(1-34) did exhibit a significant increase in ultimate stress, yield stress, and modulus compared to sedentary mice treated with vehicle.

The average post-yield toughness of all three exercise groups, at $2.3 \pm 0.2 \mathrm{MPa}$, was significantly greater than the combined average for all three sedentary groups, which was $1.7 \pm 0.2 \mathrm{MPa}$ $(p=0.035)$. This main effect of exercise on the post-yield toughness was driven by a significant interaction between exercise and sedentary mice treated with vehicle $(2.39 \pm 0.3 \mathrm{MPa}$ exercise, $1.46 \pm 0.1 \mathrm{MPa}$ sedentary; $p=0.045$ ). No significant interactions were found between groups treated with either PTH(7-34) or PTH(1-34) for pre-yield or post-yield toughness.

\section{Discussion}

Based on our findings, the systemic release of PTH during exercise provides a key signaling mechanism for both trabecular and cortical bone adaptation. Although bone adaptation in response to exercise is commonly attributed to dynamic loading, inhibition of PTH signaling during exercise via PTH(734) treatment revealed its key role in regulating bone adaptation. After 3 weeks of exercise, PTH signaling contributed to an increase in trabecular bone volume that was characterized as a more platelike structure with thicker trabeculae (Fig. 3). In addition, PTH signaling also contributed to cortical bone adaptation that resulted in increased structural-level properties 
A
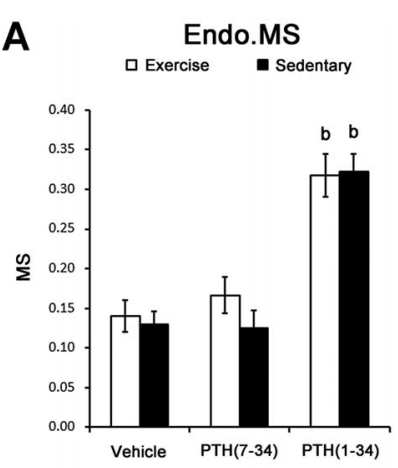

B
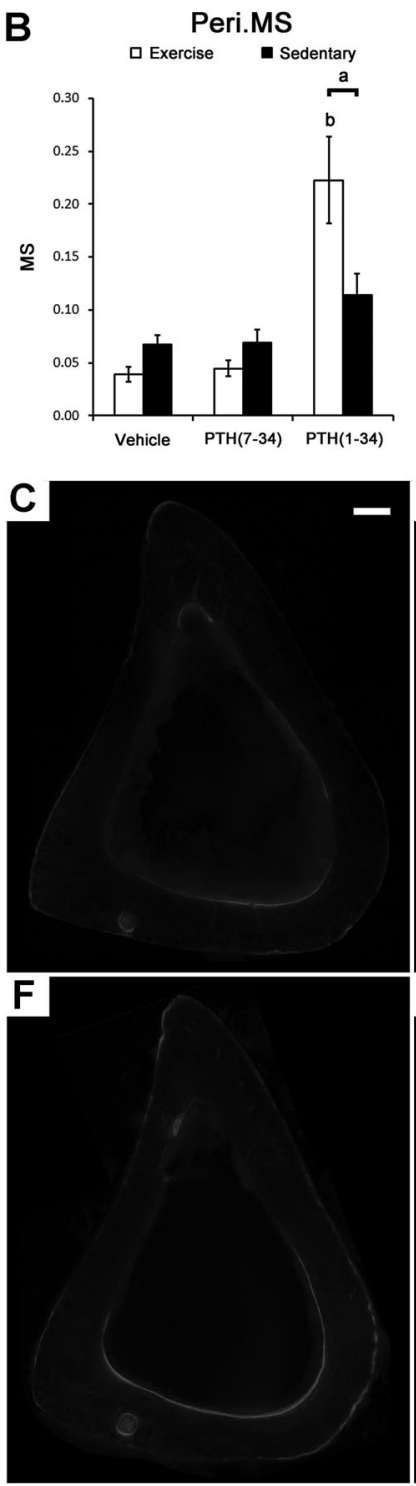

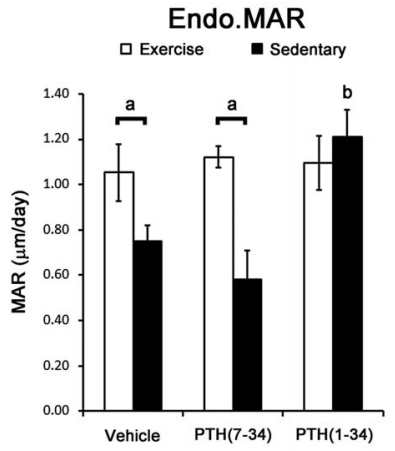

Peri.MAR

$\checkmark$ Exercise Sedentary
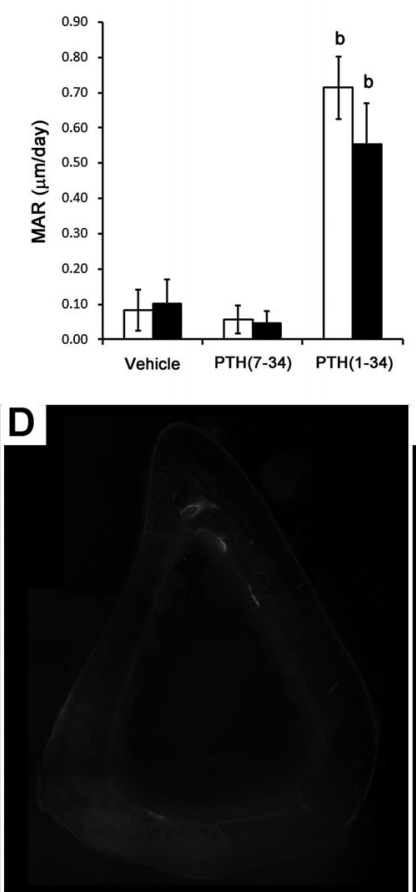

G

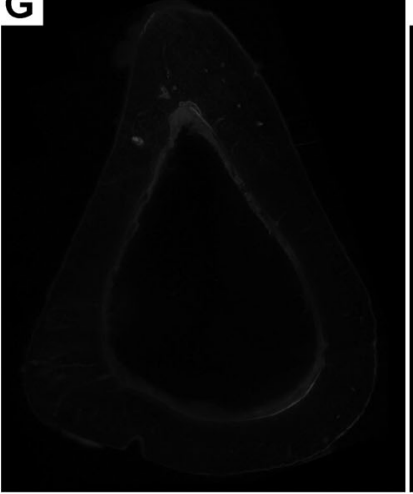

Endo.BFR

प Exercise Sedentary

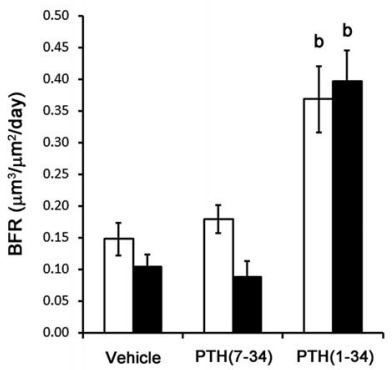

Peri.BFR
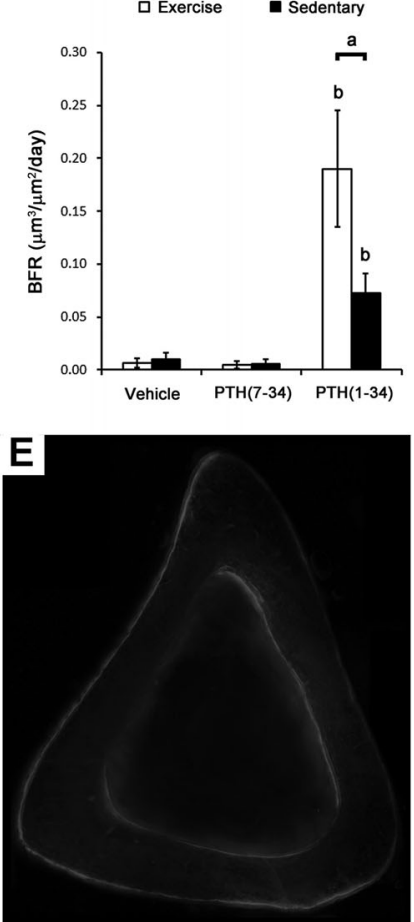

H

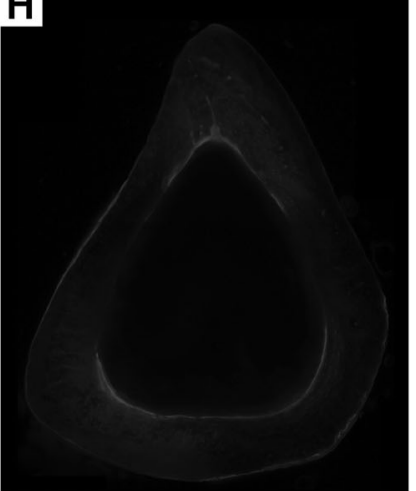

Fig. 5. Cortical bone formation increases at the endosteum and periosteum with PTH(1-34) treatment, but is unaltered by exercise. Representative images demonstrate that sedentary mice treated with vehicle $(A)$ or PTH(7-34) $(B)$ exhibited little bone formation compared to sedentary mice treated with PTH(1-34) (C). Exercise groups treated with vehicle $(D)$ and PTH(7-34) (E) also showed little bone formation compared to PTH(1-34) (F) treatment during exercise. Images shown are representative of each group of 12 samples (Bar $=100 \mu \mathrm{m})$. (G) At the endosteal surface, the mineralizing surface and BFR were significantly increased in response to PTH(1-34), whereas the MAR was significantly reduced in response to exercise. (H) At the periosteal surface, PTH(1-34) treatment alone caused a significant increase in MS, MAR, and BFR, whereas the combination of PTH(1-34) treatment and exercise significantly increased the MAR and BFR, but not the MS compared to vehicle-treated controls. Mean $\pm \mathrm{SEM}(n=12$, a indicates $p<0.05, \mathrm{~b}$ indicates $p<$ 0.05 compared to vehicle control). Endo = endosteal surface; $M S=$ mineralizing surface; $B F R=$ bone formation rate; $M A R=$ mineral apposition rate; Peri $=$ periosteal surface. 
Table 1. $\mu C T$ Analysis of Cortical Bone at the Standard Site

\begin{tabular}{|c|c|c|c|c|c|c|}
\hline & \multicolumn{3}{|c|}{ Sedentary } & \multicolumn{3}{|c|}{ Exercise } \\
\hline & Vehicle & PTH(7-34) & PTH(1-34) & Vehicle & PTH(7-34) & PTH(1-34) \\
\hline Tibia length $(\mathrm{cm})$ & $17.5 \pm 0.06$ & $17.4 \pm 0.06$ & $17.4 \pm 0.07$ & $17.4 \pm 0.06$ & $17.3 \pm 0.05$ & $17.4 \pm 0.05$ \\
\hline Ct.Ar $\left(\mathrm{mm}^{2}\right)$ & $0.746 \pm 0.016$ & $0.743 \pm 0.017$ & $0.795 \pm 0.014^{\mathrm{a}}$ & $0.728 \pm 0.019$ & $0.726 \pm 0.009$ & $0.789 \pm 0.010^{b}$ \\
\hline Ct.Th (mm) & $0.205 \pm 0.004$ & $0.208 \pm 0.003$ & $0.224 \pm 0.003^{a}$ & $0.207 \pm 0.003$ & $0.208 \pm 0.003$ & $0.220 \pm 0.002^{b}$ \\
\hline $\mathrm{D}_{\mathrm{N}-\mathrm{A}}(\mathrm{mm})$ & $0.604 \pm 0.007$ & $0.600 \pm 0.009$ & $0.608 \pm 0.010$ & $0.606 \pm 0.011$ & $0.589 \pm 0.010$ & $0.605 \pm 0.006$ \\
\hline $\mathrm{l}_{\mathrm{ap}}\left(\mathrm{mm}^{4}\right)$ & $0.093 \pm 0.004$ & $0.089 \pm 0.004$ & $0.094 \pm 0.004$ & $0.085 \pm 0.005$ & $0.082 \pm 0.002$ & $0.094 \pm 0.002$ \\
\hline $\mathrm{I}_{\mathrm{ml}}\left(\mathrm{mm}^{4}\right)$ & $0.172 \pm 0.008$ & $0.167 \pm 0.008$ & $0.181 \pm 0.006$ & $0.162 \pm 0.010$ & $0.159 \pm 0.005$ & $0.184 \pm 0.006$ \\
\hline $\mathrm{BMD}\left(\mathrm{mg} \mathrm{HA} / \mathrm{cm}^{2}\right)$ & $1200.8 \pm 6.2$ & $1210.9 \pm 5.2$ & $1202.3 \pm 8.2$ & $1204.2 \pm 5.9$ & $1215.7 \pm 5.4$ & $1202.0 \pm 3.5$ \\
\hline $\mathrm{BMC}(\mathrm{mg} \mathrm{HA})$ & $150.5 \pm 3.6$ & $151.2 \pm 3.6$ & $160.7 \pm 3.3^{a}$ & $147.3 \pm 3.7$ & $148.3 \pm 2.2$ & $159.3 \pm 2.1^{b}$ \\
\hline
\end{tabular}

Values are mean \pm SEM.

${ }^{a} p<0.05$ compared to Sedentary + Vehicle and Sedentary + PTH(7-34).

${ }^{\mathrm{b}} p<0.05$ compared to Exercise + Vehicle and Exercise + PTH(7-34).

of the tibia, demonstrated by an increase in ultimate load and stiffness without the expense of decreased yield load (Fig. 6A). In contrast, the gain in tissue-level properties following 3 weeks of exercise was independent of PTH signaling (Fig. 6B) and is mediated by another mechanism. Overall, this is the first study to demonstrate that PTH signaling during exercise contributes to trabecular and cortical bone adaptation, and that PTH signaling influences the adaptation of cortical bone at the structural level and tissue level differently.

The dynamic loading and transient increase in PTH levels during exercise provide a unique combination of stimuli, which together regulate bone adaptation. Other exercise regimens that include running and swimming have exhibited a similar increase in trabecular bone formation. ${ }^{(6,7)}$ Based on our results, the shift toward a more platelike structure during exercise was not only a function of PTH, but likely due to a combination of factors given the negligible impact of $\mathrm{PTH}(1-34)$ alone. This shift to a more platelike structure along with the change in eigenvalues is counter to the shift toward a more rodlike structure observed with aging and onset of osteoporosis. ${ }^{(30,31)}$ However, the mechanical advantage gained by this adaptation remains unclear, although the more platelike architecture has
A

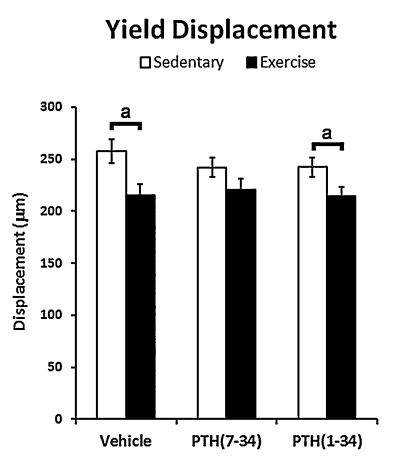

B

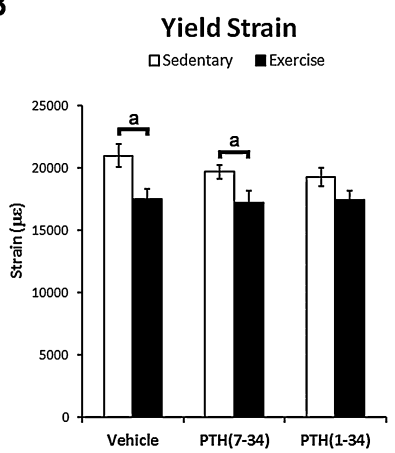

Yield Load usedentary $\mathbf{E x e r c i s e}$

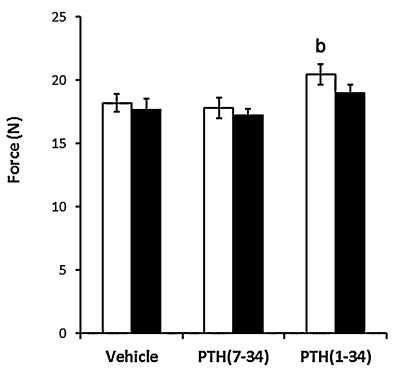

Yield Stress

口Sedentary $\mathbf{m}$ Exercise



Stiffness

口Sedentary Exercise

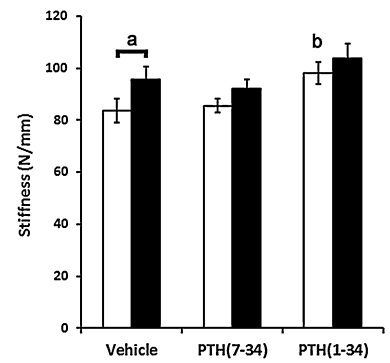

Modulus uSedentary $\mathbf{E}$ Exercise

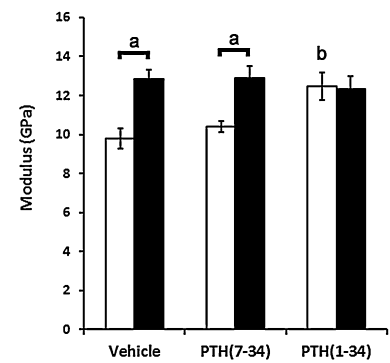

Ultimate Load usedentary Exercise

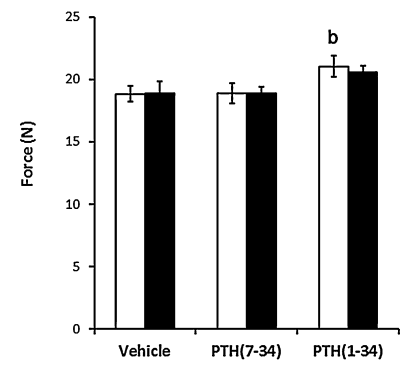

Ultimate Stress

usedentary Exercise

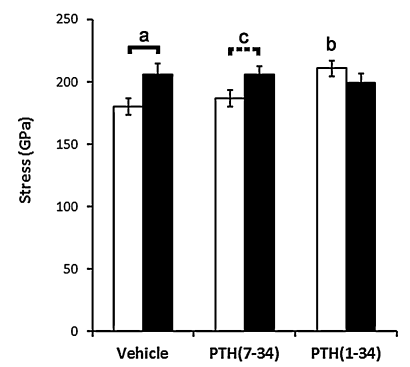

Fig. 6. PTH signaling during exercise mediates changes in structural-level mechanical properties, but not tissue-level mechanical properties of cortical bone. $(A)$ Of the structural-level mechanical properties, exercise had statistically significant influences on yield-displacement and stiffness, which were inhibited by PTH(7-34) treatment during exercise. (B) Of the tissue-level properties, exercise had a statistically significant effect on yield strain, modulus, and ultimate stress independent of PTH signaling. Mean \pm SEM ( $n=12$, a indicates $p<0.05 ; b$ indicates $p<0.05$ compared to vehicle sedentary control, $c$ indicates $p<0.055)$. 
been associated with increased toughness and resistance to microdamage. ${ }^{(32,33)}$

Similar to previous studies, exercise caused a significant increase in ultimate stress and toughness without significant changes in cortical area, mineral density, or moment of inertia (Fig. 6, Table 1). ${ }^{(10,12)}$ In contrast, the increase in cortical area and BMC following PTH(1-34) treatment corresponded with an increase in structural-level and tissue-level mechanical properties, such as stiffness and modulus (Fig. 6), similar to previous studies. ${ }^{(34,35)}$ Although exercise did not change the quantity of tissue being formed (Fig. 5, Table 1), mechanical loading and PTH can enhance osteoblast expression of collagenous and noncollagenous proteins that have distinct influences over the ensuing mechanical properties. ${ }^{(36,37)}$ Exercise can modify the intrinsic tissue properties of cortical bone by altering the carbonate to phosphate ratio, mineral to matrix ratio, and crystallinity, as well as the ratio between mature and immature collagen cross-linking. ${ }^{(10,14,38,39)}$ Thus, we suspect that the matrix formed during exercise is being modified, and it would prove useful for future studies to identify the physiological stimuli that mediate this response.

A unique finding from this study was the attenuation in periosteal bone formation during exercise despite the addition of PTH(1-34) (Fig. 5H). Under sedentary conditions, treatment with $\mathrm{PTH}(1-34)$ caused an increase in periosteal BFR from an increase in mineralizing surface and apposition rate, similar to previous studies. ${ }^{(34,35,40,41)}$ Although the addition of PTH(1-34) during exercise significantly increased the periosteal BFR compared to exercise alone, it was significantly less compared to PTH(1-34) treatment of sedentary mice because of a significant decrease in the mineralizing surface (Fig. $5 H$ ). The typical increase in mineralizing surface by $\mathrm{PTH}(1-34)$ at the periosteum could be counteracted during exercise by a reduction in calcium availability due to an increased demand associated with muscle activity or development along the periosteum. ${ }^{(42,43)}$ When muscle demand for calcium is a limiting factor for mineralization at the periosteum, increasing the available calcium may enhance this response. Although calcium supplementation alongside exercise can increase the BMD at the femoral neck, ${ }^{(44)}$ its impact on mineralization at the periosteum is unclear. In addition, the use of calcium supplements must be carefully assessed to ensure they do not impair the systemic release of PTH during exercise, which occurs in response to a decrease in calcium reabsorption through the kidney. ${ }^{(24,45)}$

Increasing the levels of PTH during exercise via PTH(1-34) treatment superimposed the effects of $\mathrm{PTH}(1-34)$ onto those of exercise to regulate the adaptation of trabecular architecture. The increase in Tb.Th from PTH(1-34) treatment corresponded with an increase in Tb.Sp, similar to other studies that have attributed this type of response to the fusion of adjacent trabeculae as they grow thicker, which in turn explains the decrease in Tb.N. ${ }^{(46,47)}$ Although a moderate increase in trabecular BV/TV was achieved with the addition of PTH(1-34) treatment during exercise (Fig. 3), it was not significantly greater compared to exercise or PTH(1-34) treatment alone. Similarly, PTH(1-34) treatment during exercise did not enhance the changes in structural-level or tissue-level mechanical properties normally gained in response to exercise or PTH(1-34) individually (Fig. 6). In contrast, treating with PTH(1-34) 30 to 45 min prior to dynamic loading has an additive effect on trabecular and cortical bone formation that is greater than the individual effect of $\mathrm{PTH}(1-34)$ or dynamic loading. ${ }^{(25,26)}$ As a result, the lack of significance or additive effect from PTH(1-34) observed in our study is considered a function of timing in PTH(1-34) treatment. Initiating the cellular response to PTH 30 or $45 \mathrm{~min}$ prior to exercise has the potential to increase BV/TV or enhance the mechanical properties beyond what we observed by treating only 15 min prior to exercise. ${ }^{(25,26)}$ Overall, $\mathrm{PTH}(1-34)$ treatment $15 \mathrm{~min}$ prior to exercise provided a means to increase bone quantity, which can have long-term benefits, whereas additional studies are needed to establish the appropriate timing of PTH(134) that will optimize its impact on bone adaptation. In addition, the lack of change in cortical area and BFR during exercise indicates that the primary action of PTH released during exercise is not the same as $\mathrm{PTH}(1-34)$.

At the whole-bone level, exercise caused significant changes in both structural-level and tissue-level mechanical properties (Fig. 6), without significant changes in cortical bone geometry, $\mathrm{BMD}$, or BFR (Fig. 5). Under the same exercise regimen, different age mice undergo similar changes in yield deformation and ultimate stress, ${ }^{(10-12)}$ whereas younger mice exhibit additional changes in yield load, cortical area, and BMD. ${ }^{(11,14)}$ With increasing age, bone adaptation in response to exercise is significantly diminished. ${ }^{(48)}$ In addition to age, the intensity of exercise can also have a significant effect on bone adaptation. ${ }^{(49)}$ As a result, it may prove useful to investigate if increasing the intensity of exercise would increase cortical area in addition to providing the changes in mechanical properties observed in our study.

One of the limitations in this study was the inability to distinguish which source of PTH was mediating the adaptation in cortical and trabecular bone during exercise. PTH secreted by the parathyroid gland, along with the PTH-related protein (PTH$\mathrm{rP})$ secreted within bone or other tissues have the ability to activate the PTH/PTH-rP receptor (PPR). ${ }^{(50)}$ The transient increase in systemic levels of PTH measured during a single bout of exercise (Fig. 1) represents the 84-amino acid sequence of intact PTH secreted by the parathyroid gland. At the cellular level, PTH and PTH-rP have the same affinities for the PPR due to structural similarities within the 1-13 and 29-34 amino acid sequences. ${ }^{(50)}$ As a result, the available PTH in bone during exercise is underrepresented by the systemic measurements of PTH conducted here. Despite this limitation, we demonstrated that bone adaptation in response to exercise is influenced by PTH-mediated or PTH-rP-mediated signaling. The use of PTH(734) to inhibit the cellular response to PTH or PTH-rP in bone has been widely used and demonstrated to not alter systemic PTH levels or urinary secretion of calcium, phosphorus, and adenosine $3^{\prime}, 5^{\prime}$-cyclic monophosphate. ${ }^{(51-54)}$

In conclusion, exercise subjects bone to increased dynamic loading and PTH levels, both of which are key stimulants for bone adaptation. In this study we demonstrated that PTH signaling during exercise increases trabecular bone formation with a platelike structure and improves structural-level mechanical properties of cortical bone, whereas tissue-level properties are modified independent of PTH signaling during exercise. In addition, we found that $\mathrm{PTH}(1-34)$ treatment during exercise increases trabecular bone formation, along with periosteal and endosteal cortical bone formation beyond what exercise alone provides. Overall, the distinctive increase in PTH levels during exercise plays a key role in bone adaptation, specifically trabecular bone formation and the structural-level properties of cortical bone, whereas PTH(1-34) can be used to improve cortical bone formation with superior structural-level and tissuelevel mechanical properties. 


\section{Disclosures}

All authors state that they have no conflicts of interest.

\section{Acknowledgments}

This work was supported by grants from NIH/NIDCR DE07057 (to DHK); NIH/NIAMS AR056657 (to DHK); and NIH/NIAMS AR064668 (to JDG).

Authors' roles: Study design: JG and DK. Study conduct: JG and FM. Data collection: JG and FM. Data analysis: JG and FM. Data interpretation: JG, FM, and DK. Drafting manuscript: JG. Revising manuscript content: JG, FM and DK. Approving final version of manuscript: JG and DK. JG and DK take responsibility for the integrity of the data analysis.

\section{References}

1. Johnell $O$, Kanis JA. An estimate of the worldwide prevalence and disability associated with osteoporotic fractures. Osteoporos Int. 2006;17(12):1726-33.

2. Marie PJ, Kassem M. Osteoblasts in osteoporosis: past, emerging, and future anabolic targets. Eur J Endocrinol. 2011;165(1):1-10.

3. Wallace BA, Cumming RG. Systematic review of randomized trials of the effect of exercise on bone mass in pre- and postmenopausal women. Calcif Tissue Int. 2000;67(1):10-8.

4. Scott JPR, Sale C, Greeves JP, Casey A, Dutton J, Fraser WD. The role of exercise intensity in the bone metabolic response to an acute bout of weight-bearing exercise. J Appl Physiol. 2011;110(2):423-32.

5. Maimoun L, Simar D, Caillaud C, et al. Response of calciotropic hormones and bone turnover to brisk walking according to age and fitness level. J Sci Med Sport. 2009;12(4):463-7.

6. Joo $Y$, Sone $T$, Fukunaga $M$, Onodera $S$. Effects of endurance treadmill exercise on bone mineral density and bone microarchitecture in the young growing rat: Microcomputed tomography and dual energy x-ray absorptiometry study. Osteoporos Int. 2000;11:44-5.

7. Warner SE, Shea JE, Miller SC, Shaw JM. Adaptations in cortical and trabecular bone in response to mechanical loading with and without weight bearing. Calcif Tissue Int. 2006;79(6):395-403.

8. Huang TH, Lin SC, Chang FL, Hsieh SS, Liu SH, Yang RS. Effects of different exercise modes on mineralization, structure, and biomechanical properties of growing bone. J Appl Physiol. 2003;95(1): 300-7.

9. Iwamoto J, Shimamura C, Takeda T, et al. Effects of treadmill exercise on bone mass, bone metabolism, and calciotropic hormones in young growing rats. J Bone Miner Metab. 2004;22(1):26-31.

10. Kohn DH, Sahar ND, Wallace JM, Golcuk K, Morris MD. Exercise alters mineral and matrix composition in the absence of adding new bone. Cells Tissues Organs. 2009;189(1-4):33-7.

11. Wallace JM, Rajachar RM, Allen MR, et al. Exercise-induced changes in the cortical bone of growing mice are bone- and gender-specific. Bone. 2007:40(4):1120-7.

12. Wallace JM, Ron MS, Kohn DH. Short-term exercise in mice increases tibial post-yield mechanical properties while two weeks of latency following exercise increases tissue-level strength. Calcif Tissue Int. 2009;84(4):297-304.

13. Iwamoto J, Yeh JK, Aloia JF. Differential effect of treadmill exercise on three cancellous bone sites in the young growing rat. Bone. 1999; 24(3):163-9.

14. Wallace JM, Golcuk K, Morris MD, Kohn DH. Inbred strain-specific effects of exercise in wild type and biglycan deficient mice. Ann Biomed Eng. 2010;38(4):1607-17.

15. Burr DB, Milgrom C, Fyhrie D, et al. In vivo measurement of human tibial strains during vigorous activity. Bone. 1996;18(5):405-10.

16. Brodt MD, Silva MJ. Aged mice have enhanced endocortical response and normal periosteal response compared with young- adult mice following 1 week of axial tibial compression. J Bone Miner Res. 2010;25(9):2006-15.

17. Mosley JR, Lanyon LE. Strain rate as a controlling influence on adaptive modeling in response to dynamic loading of the ulna in growing male rats. Bone. 1998;23(4):313-8.

18. Robling AG, Castillo $A B$, Turner $\mathrm{CH}$. Biomechanical and molecular regulation of bone remodeling. Annu Rev Biomed Eng. 2006;8: 455-98.

19. Chen XY, Zhang XZ, Guo Y, Li RX, Lin JJ, Wei Y. The establishment of a mechanobiology model of bone and functional adaptation in response to mechanical loading. Clin Biomech. 2008;23:S88-95.

20. De Souza RL, Matsuura M, Eckstein F, Rawlinson SCF, Lanyon LE, Pitsillides AA. Non-invasive axial loading of mouse tibiae increases cortical bone formation and modifies trabecular organization: a new model to study cortical and cancellous compartments in a single loaded element. Bone. 2005;37(6):810-8.

21. Holguin N, Brodt MD, Sanchez ME, Kotiya AA, Silva MJ. Adaptation of tibial structure and strength to axial compression depends on loading history in both $\mathrm{C} 57 \mathrm{BL} / 6$ and BALB/c mice. Calcif Tissue Int. 2013;93(3):211-21.

22. Robling AG, Hinant FM, Burr DB, Turner $\mathrm{CH}$. Improved bone structure and strength after long-term mechanical loading is greatest if loading is separated into short bouts. J Bone Miner Res. 2002;17(8): 1545-54.

23. Maimoun L, Sultan C. Effect of physical activity on calcium homeostasis and calciotropic hormones: a review. Calcif Tissue Int. 2009;85(4):277-86.

24. Brahm H, PiehlAulin $\mathrm{K}$, Ljunghall S. Bone metabolism during exercise and recovery: the influence of plasma volume and physical fitness. Calcif Tissue Int. 1997;61(3):192-8.

25. Sugiyama T, Saxon LK, Zaman G, et al. Mechanical loading enhances the anabolic effects of intermittent parathyroid hormone (1-34) on trabecular and cortical bone in mice. Bone. 2008;43(2):238-48.

26. Chow JWM, Fox S, Jagger CJ, Chambers TJ. Role for parathyroid hormone in mechanical responsiveness of rat bone. Am J Physiol Endocrinol Metab. 1998;274(1):E146-54.

27. McCauley LK, Koh AJ, Beecher CA, Rosol TJ. Proto-oncogene c-fos is transcriptionally regulated by parathyroid hormone (PTH) and PTH-related protein in a cyclic adenosine monophosphatedependent manner in osteoblastic cells. Endocrinology. 1997; 138(12):5427-33.

28. Cowin SC. The relationship between the elasticity tensor and the fabric tensor. Mech Mater. 1985;4(2):137-47.

29. Dempster DW, Compston JE, Drezner MK, et al. Standardized nomenclature, symbols, and units for bone histomorphometry: a 2012 update of the report of the ASBMR Histomorphometry Nomenclature Committee. J Bone Miner Res. 2013 Jan;28(1):2-17.

30. Ding $M$, Hvid I. Quantification of age-related changes in the structure model type and trabecular thickness of human tibial cancellous bone. Bone. 2000;26(3):291-5.

31. Brouwers JEM, Lambers FM, Gasser JA, van Rietbergen B, Huiskes $R$. Bone degeneration and recovery after early and late bisphosphonate treatment of ovariectomized Wistar rats assessed by in vivo micro-computed tomography. Calcif Tissue Int. 2008;82(3):202-11.

32. Garrison JG, Slaboch CL, Niebur GL. Density and architecture have greater effects on the toughness of trabecular bone than damage. Bone. 2009;44(5):924-9.

33. Teo JCM, Si-Hoe KM, Keh JEL, Teoh SH. Relationship between CT intensity, micro-architecture and mechanical properties of porcine vertebral cancellous bone. Clin Biomech. 2006;21(3):235-44.

34. Ejersted $\mathrm{C}$, Andreassen $\mathrm{T}$, Nilsson MHL, Oxlund $\mathrm{H}$. Human parathyroid hormone(1-34) increases bone-formation and strength of cortical bone in aged rats. Eur J Endocrinol. 1994;130(2):201-7.

35. Hirano T, Burr DB, Turner CH, Sato M, Cain RL, Hock JM. Anabolic effects of human biosynthetic parathyroid hormone fragment (1-34), LY3333334, on remodeling and mechanical properties of cortical bone in rabbits. J Bone Miner Res. 1999;14(4):536-45.

36. Swarthout JT, D'Alonzo RC, Selvamurugan N, Partridge NC. Parathyroid hormone-dependent signaling pathways regulating genes in bone cells. Gene. 2002;282(1-2):1-17. 
37. Papachroni KK, Karatzas DN, Papavassiliou KA, Basdra EK, Papavassiliou AG. Mechanotransduction in osteoblast regulation and bone disease. Trends Mol Med. 2009;15(5):208-16.

38. Follet $H$, Boivin G, Rumelhart C, Meunier PJ. The degree of mineralization is a determinant of bone strength: a study on human calcanei. Bone. 2004;34(5):783-9.

39. Morris MD, Mandair GS. Raman assessment of bone quality. Clin Orthop Relat Res. 2011;469(8):2160-9.

40. lida-Klein A, Lu SS, Cosman F, Lindsay R, Dempster DW. Effects of cyclic vs. daily treatment with human parathyroid hormone (1-34) on murine bone structure and cellular activity. Bone. 2007; 40(2):391-8.

41. Zhou $\mathrm{H}$, lida-Klein A, Lu SS, et al. Anabolic action of parathyroid hormone on cortical and cancellous bone differs between axial and appendicular skeletal sites in mice. Bone. 2003;32(5):513-20.

42. Frost HM, Schonau E. The "muscle-bone unit" in children and adolescents: a 2000 overview. J Pediatr Endocrinol Metab. 2000;13 (6):571-90.

43. Wassner SJ, Li JB, Sperduto A, Norman ME. Vitamin-D deficiency, hypocalcemia, and increased skeletal-muscle degradation in rats. J Clin Invest. 1983;72(1):102-12.

44. Prince R, Devine A, Dick $\mathrm{l}$, et al. The effects of calcium supplementation (milk powder or tablets) and exercise on bonedensity in postmenopausal women. J Bone Miner Res. 1995;10(7): 1068-75.

45. Ashizawa N, Fujimura R, Tokuyama K, Suzuki M. A bout of resistance exercise increases urinary calcium independently of osteoclastic activation in men. J Appl Physiol. 1997;83(4):1159-63.
46. Lynch MA, Brodt MD, Stephens AL, Civitelli R, Silva MJ. Lowmagnitude whole-body vibration does not enhance the anabolic skeletal effects of intermittent PTH in adult mice. J Orthop Res. 2011;29(4):465-72.

47. Sheehan S, Muthusamy A, Paul E, Sikes RA, Gomes RR. Short-term intermittent PTH 1-34 administration enhances bone formation in SCID/Beige mice. Endocr J. 2010;57(5):373-82.

48. Hoshi A, Watanabe $H$, Chiba $M$, Inaba $Y$. Effects of exercise at different ages on bone density and mechanical properties of femoral bone of aged mice. Tohoku J Exp Med. 1998;185(1): $15-24$.

49. Peng ZQ, Vaananen HK, Tuukkanen J. Ovariectomy-induced bone loss can be affected by different intensities of treadmill running exercise in rats. Calcif Tissue Int. 1997;60(5):441-8.

50. Esbrit $\mathrm{P}$, Alcaraz MJ. Current perspectives on parathyroid hormone (PTH) and PTH-related protein (PTHrP) as bone anabolic therapies. Biochem Pharmacol. 2013;85(10):1417-23.

51. Rosen HN, Lim M, Garber J, et al. The effect of PTH antagonist BIM44002 on serum calcium and PTH levels in hypercalcemic hyperparathyroid patients. Calcif Tissue Int. 1997;61(6):455-9.

52. Caulfield MP, Rosenblatt M. Parathyroid-hormone receptor interactions. Trends Endocrinol Metab. 1990;1(3):164-8.

53. Goldman ME, Mckee RL, Caulfield MP, et al. A new highly potent parathyroid-hormone antagonist - [D-Trp12, Tyr34]Bpth-(7-34) Nh2. Endocrinology. 1988;123(5):2597-9.

54. Horiuchi N, Hongo T, Clemens TL. A 7-34 analog of the parathyroid hormone-related protein has potent antagonist and partial agonist activity in vivo. Bone Mineral. 1991;12(3):181-88. 